\title{
Clinical Practice Guidelines for Fecal Microbiota Transplantation in Korea
}

\author{
Tae-Geun Gweon, ${ }^{1}$ Yoo Jin Lee, ${ }^{2}$ Kyeong Ok Kim, ${ }^{3}$ Sung Kyun Yim, ${ }^{4}$ Jae Seung Soh, ${ }^{5}$ Seung Young Kim, ${ }^{6}$ Jae Jun Park, \\ Seung Yong Shin, ${ }^{8}$ Tae Hee Lee, ${ }^{9}$ Chang Hwan Choi, ${ }^{8}$ Young-Seok Cho, ${ }^{1 *}$ Dongeun Yong, ${ }^{10}$ Jin-Won Chung, ${ }^{11}$ Kwang Jae Lee, ${ }^{12}$ \\ Oh Young Lee, ${ }^{13}$ Myung-Gyu Choi, ${ }^{1}$ and Miyoung Choi ${ }^{14}$; Gut Microbiota and Therapy Research Group Under the Korean Society \\ of Neurogastroenterology and Motility
}

${ }^{1}$ Department of Internal Medicine, College of Medicine, The Catholic University of Korea, Seoul, Korea; ${ }^{2}$ Department of Internal Medicine, Keimyung University School of Medicine, Daegu, Korea; ${ }^{3}$ Division of Gastroenterology and Hepatology, Department of Internal Medicine, Yeungnam University College of Medicine, Daegu, Korea; ${ }^{4}$ Division of Gastroenterology and Hepatology, Department of Internal Medicine, Jeonbuk National University Medical School and Hospital, Jeonju, Jeollabuk-do, Korea; ${ }^{5}$ Division of Gastroenterology and Hepatology, Department of Internal Medicine, University of Hallym College of Medicine, Hallym University, Anyang, Gyeonggi-do, Korea; ${ }^{6}$ Division of Gastroenterology and Hepatology, Department of Internal Medicine, Jeonbuk National University Medical School, Jeonju, Jeollabuk-do, Korea; ${ }^{7}$ Division of Gastroenterology and Hepatology, Department of Internal Medicine, Yonsei University College of Medicine, Seoul, Korea; ${ }^{8}$ Division of Gastroenterology and Hepatology, Department of Internal Medicine, Chung-Ang University College of Medicine, Seoul, Korea; ${ }^{9}$ Institute for Digestive Research, Digestive Disease Center, Soonchunhyang University College of Medicine, Seoul, Korea; ${ }^{10}$ Department of Laboratory Medicine and Research Institute of Bacterial Resistance, Yonsei University College of Medicine, Seoul, Korea; ${ }^{11}$ Division of Infectious Diseases, Department of Internal Medicine, Chung-Ang University College of Medicine, Seoul, Korea; ${ }^{12}$ Department of Internal Medicine, Ajou University School of Medicine, Suwon, Gyeonggi-do, Korea; ${ }^{13}$ Department of Internal Medicine, Hanyang University School of Medicine, Seoul, Korea; and ${ }^{14}$ Division of Healthcare Technology Assessment Research, National Evidence-Based Healthcare Collaboration Agency, Seoul, Korea

Fecal microbiota transplantation (FMT) is a highly efficacious and safe modality for the treatment of recurrent or refractory Clostridioides difficile infection (CDI), with overall success rates of $90 \%$. Thus, FMT has been widely used for 10 years. The incidence and clinical characteristics of CDI, the main indication for FMT, differ between countries. To date, several guidelines have been published. However, most of them were published in Western countries and therefore cannot represent the Korean national healthcare systems. One of the barriers to performing FMT is a lack of national guidelines. Accordingly, multidisciplinary experts in this field have developed practical guidelines for FMT. The purpose of these guidelines is to aid physicians performing FMT, which can be adapted to treat $\mathrm{CDI}$ and other conditions.

(J Neurogastroenterol Motil 2022;28:28-42)

\section{Key Words}

Fecal microbiota transplantation; Guideline; Treatment

Received: November 16, 2021 Revised: None Accepted: December 8, 2021

(.) This is an Open Access article distributed under the terms of the Creative Commons Attribution Non-Commercial License (http://creativecommons. org/licenses/by-nc/4.0) which permits unrestricted non-commercial use, distribution, and reproduction in any medium, provided the original work is properly cited.

*Correspondence: Young-Seok Cho, MD, PhD Division of Gastroenterology, Department of Internal Medicine, The Catholic University of Korea, Seoul St. Mary's Hospital, 222 Banpo-daero, Seocho-gu, Seoul 06591, Korea

Tel: +82-2-2258-6021, Fax: +82-2-2258-2038, E-mail: yscho@catholic.ac.kr

Tae-Geun Gweon and Yoo Jin Lee equally contributed to this article. 


\section{Introduction}

Fecal microbiota transplantation (FMT) is a highly efficacious and safe modality for the treatment of recurrent or refractory Clostridioides difficile infection (CDI), with overall success rates of 90\%. With the great success of FMT for CDI and the development of next-generation sequencing, FMT has been tested for a range of other diseases including metabolic and gastrointestinal (GI) diseases such as irritable bowel syndrome (IBS) and inflammatory bowel disease (IBD), and several guidelines have been published. ${ }^{2-6}$ Although FMT has been tried for non-CDI diseases, the methods and results are mixed. While previous guidelines focused on FMT for the treatment of CDI, both disease prevalence and healthcare systems are heterogenous across countries. Korean FMT guidelines are needed because the lack of national guidelines is one of the major hurdles for FMT. In the guidelines developed here, we focus on the practical aspects of FMT, rather than on indication and basis.

\section{Methods}

\section{Direction}

The process of FMT includes donor screening, production of stool material, and delivery. Therefore, multidisciplinary expertise is involved in the development of these guidelines. Members of the Gut Microbiota and Therapy Research Group of the Korean Society of Neurogastroenterology and Motility (KSNM) are mainly involved in developing these guidelines. Multi-academic societies, including the Korean Society of Gastroenterology, the Korean Society of Infectious Diseases, and the Korean Society for Laboratory Medicine, participated as well. The working group consisted of 11 gastroenterologists, 1 professor of infectious diseases, 1 professor of laboratory medicine, and 1 methodologist invited from the National Evidence-based Healthcare Collaborating Agency. Six workshops were held for all working participants. The first meeting took place on July 31, 2019. After the outbreak of the novel coronavirus disease 2019 (COVID-19), 4 of the 6 meetings were held online.

\section{Development Process}

The working group decided to follow the adaptation method for guidelines development. Seven guidelines were assessed for suitability for adaptation using the Korean Appraisal of Guidelines for Research and Evaluation II. ${ }^{7}$ After rigorous review, 5 guidelines were included for adaptation (Table 1 ). ${ }^{2-6}$ For recent articles, MEDLINE, Embase, and Web of Science were searched for literature that were published after the reference guidelines between January 2017 and September 2020. Human clinical trials were mainly extracted. For quality assessment, the RoB 1.0 and RoBANS 2.0 tools were used for randomized controlled trials (RCTs) and observational studies, respectively. ${ }^{8}$ Efficacy and safety should be considered together for the practical aspects of FMT. Optimal FMT practice should be done to increase efficacy and minimize adverse events (AEs). For CDI, the efficacy of the first FMT was reported to be about $80 \%$. The overall efficacy of FMT can be increased by the performance of multiple FMTs. ${ }^{9,10}$ However, this is accompanied by increased medical costs, inconvenience for the patient, and the possibility of AEs. Therefore, physicians should try to enhance the success rate of the first FMT. Since FMT has become widespread for various indications, several fatal AEs have been reported to date regarding endoscopy-related complications and infectious complications. We emphasize the safety issue of FMT in these guidelines. Case reports or conference abstracts were included regarding fatalities in cases of FMT.

Table 1. Selected Guidelines for Adaptation

\begin{tabular}{|c|c|c|c|c|}
\hline Author & Title & Country & Journal & Year \\
\hline Cammarota et $\mathrm{al}^{2}$ & European consensus conference on fecal microbiota transplantation in clinical practice & Europe & Gut & 2017 \\
\hline Mullish et $\mathrm{al}^{3}$ & $\begin{array}{l}\text { The use of fecal microbiota transplant as treatment for recurrent or refractory } \\
\text { Clostridium difficile infection and other potential indications: joint British Society of } \\
\text { Gastroenterology and Healthcare Infection Society guidelines }\end{array}$ & United Kingdom & Gut & 2018 \\
\hline Cammarota et $\mathrm{al}^{4}$ & $\begin{array}{l}\text { International consensus conference on stool banking for fecal microbiota } \\
\text { transplantation in clinical practice }\end{array}$ & International & Gut & 2019 \\
\hline $\mathrm{Ng}$ et $\mathrm{al}^{5}$ & $\begin{array}{l}\text { Scientific frontiers in fecal microbiota transplantation: joint document of Asia-Pacific } \\
\text { Association of Gastroenterology and Asia-Pacific Society for Digestive Endoscopy }\end{array}$ & Asia-Pacific & Gut & 2020 \\
\hline Haifer et $\mathrm{al}^{6}$ & $\begin{array}{l}\text { Australian consensus statements for the regulation, production and use of fecal } \\
\text { microbiota transplantation in clinical practice }\end{array}$ & Australia & Gut & 2020 \\
\hline
\end{tabular}


The guidelines and article assessments were performed by 2 independent members. In total, 103 articles were selected for the resulting guideline. The working group formulated 18 statements regarding methodology for FMT according to the quality of evidence and grade of recommendation (Table 2). Because there are few experts in this topic, the Delphi method was not adopted. Instead, grade of recommendation was formulated through voting by members of the working group.

\section{Fecal Microbiota Transplantation Indications}

\section{Statement 1: Fecal microbiota transplantation is rec- ommended for patients with recurrent Clostridioides difficile infection who have had at least 2 recurrences and can be considered in patients with refractory or severe Clostridioides difficile infection.}

(Strong recommendation, high quality of evidence)

Guidelines from the American College of Gastroenterology in 2013 and Infectious Diseases Society of America and Society for Healthcare Epidemiology of America in 2018 recommend FMT for patients with second or subsequent recurrences of CDI who have failed appropriate antibiotic therapy. ${ }^{11,12}$ In Europe and Australia, guidelines or consensus statements strongly recommend FMT for the treatment of multiple recurrent CDI, and recommend FMT for refractory or severe CDI not responding to conventional antibiotic treatment. ${ }^{2-4,6}$ We recommend FMT for recurrent CDI of at least 2 episodes. For refractory or severe CDI, FMT can be considered at the physician's discretion. To date, reports of efficacy of FMT for non-CDI diseases are mixed. When FMT is conducted for non-CDI diseases, it should be performed under regulation or for the purposes of a clinical trial.

\section{Inflammatory Bowel Disease}

Statement 2: When fecal microbiota transplantation
is performed in patients with inflammatory bowel
disease, either for Clostridioides difficile infection or
IBD treatment, a warning of inflammatory bowel dis-
ease flare should be issued.
(Strong recommendation, high quality of evidence)

IBD is a chronic and intractable intestinal disorder. Although the causal relationship has yet to be determined, dysbiosis has been proved in patients with IBD. FMT has been tried in patients with IBD for the treatment of CDI or IBD itself. ${ }^{13-16} \mathrm{~A}$ recent population-based study reported that patients with underlying IBD had a 4.8-fold higher risk of developing CDI than individuals without IBD. The risk of CDI in IBD patients is especially increased among young patients and within the first year of diagnosis. ${ }^{17} \mathrm{~A}$ systematic review reported that the mortality of CDI was higher in IBD patients compared with non-IBD patients (odds ratio [OR], 4.39 ; $95 \%$ confidence interval $\left.[\mathrm{CI}], 3.56-5.42 ; I^{2}=93 \%\right) .{ }^{18}$ Studies suggest that FMT is an effective option for the treatment of CDI in patients with underlying IBD. A recent systematic review of 9 cohort studies reported that the initial cure rate was $81 \%$, and that the overall cure rate after repeated FMT infusions was $89 \%$, suggesting that FMT is an effective treatment for recurrent CDI in individuals with underlying IBD. ${ }^{19}$ A cohort study including 272 patients undergoing FMT for recurrent CDI showed that FMT is less effective for treating recurrent CDI in IBD patients compared with those without IBD $(74.4 \%$ vs $92.1 \%, P=0.018){ }^{20}$

Some patients with IBD have been reported to have disease flare after FMT. A large retrospective cohort study, including 67 patients, showed that a minority of patients $(13 \%)$ had worsening

Table 2. Strength of Recommendation and Quality of Evidence

\begin{tabular}{|c|c|}
\hline $\begin{array}{l}\text { Strength of recommendation } \\
\text { Strong } \\
\text { Conditional }\end{array}$ & $\begin{array}{l}\text { Position } \\
\text { Most patients should receive the recommended course of action. } \\
\text { Different choices would be appropriate for different patients. }\end{array}$ \\
\hline Quality of evidence & Definition \\
\hline High & We are very confident that the true effect lies close to the estimate of the effect. \\
\hline Moderate & $\begin{array}{l}\text { We are moderately confident in the effect estimate. The true effect is likely to be close to the estimate of effect, } \\
\text { but there is a possibility that it is substantially different. }\end{array}$ \\
\hline Low & $\begin{array}{l}\text { Our confidence in the estimate is limited. The true effect may be substantially different from the estimate of ef- } \\
\text { fect. }\end{array}$ \\
\hline Very low & $\begin{array}{l}\text { We have very little confidence in the effect estimate. The true effect is likely to be substantially different from the } \\
\text { estimate of effect. }\end{array}$ \\
\hline
\end{tabular}


of disease. ${ }^{21}$ A systematic review, including 29 studies of 514 IBD patients, reported a pooled rate of IBD flare after FMT of $14.9 \%{ }^{22}$ In subgroup analysis, the rate of FMT flare was $22.7 \%$ (95\% CI, 13-36\%) in patients with CDI together with IBD, and $11.1 \%$ (95\% CI, 7-17\%) in patients with IBD alone. The results of a prospective study may be different from those of retrospective studies. A recent prospective multicenter study including 50 patients reported that disease activity in IBD after FMT was improved in 33 (67\%), no change in $15(31 \%)$, and de novo flare in $1(2 \%)$, suggesting that IBD outcomes after FMT are better than reported in retrospective studies. ${ }^{23}$ Physicians should be aware of a possibility of IBD flare after FMT, and patients should be informed of this risk.

\section{Donor Screening}

\section{Statement 3: Stool donors should be assessed for gen- eral health status and gastrointestinal conditions by clinical assessment, and serological and fecal tests. \\ (Strong recommendation, low quality of evidence) \\ Statement 4: Stool donors should repeatedly undergo the same clinical assessment, and serological and fecal tests every 2-3 months. \\ (Conditional recommendation, low quality of evidence)}

Donor screening is one of the most important steps for FMT. Stool can be obtained from a patient's related donor or an unrelated donor (universal healthy donor). All donors should be approved for stool donation and this process should be done voluntarily. Donor candidates should be fully informed of the benefits and harms of the donation and complete a written consent form. Donor's infectious, metabolic, and other pathologic conditions can be transferred to recipients. Therefore, a screening process including a clinical assessment, and serological and fecal tests should be carried out. Establishment of a non-profit stool bank has contributed to the standardization of donor screening. In 2019, international guideline regarding donor screening was published. ${ }^{4}$ It is important to note that endemic diseases differ among countries. Tests for endemic diseases that are specific to a region should be included along with common items for donor screening. High-risk countries include regions at high risk of communicable disease/traveler's diarrhea (eg, South Asia, Africa, and the Middle East) and CreutzfeldtJakob disease (United Kingdom and Europe). In Korea, where the prevalence of gastric cancer is high, donors should be screened for Helicobacter pylori infection. Donors with a recent travel history to a specific region should be screened for region-specific endemic diseases.

The first step toward donor screening is an interview for suitability for donation. Three key issues regarding the selection of potential donors are as follows: (1) known history or risk factors for infectious diseases, (2) disorders potentially associated with perturbation of gut microbiota, and (3) drugs that can alter gut microbiota. A questionnaire includes demographic characteristics, medical condition, and recent travel history. The donor screening process, which was carried out in a single Korean center showed that $74 \%$ of candidates were excluded based on this questionnaire, ${ }^{24}$ which was similar to data presented by other countries. ${ }^{25,26}$ The questionnaire should be administered during screening and on the day of stool donation. ${ }^{2,4}$ Even individuals who passed screening cannot donate their stool if newly developed conditions are revealed by the questionnaire on the day of stool collection. The items for questionnaire and laboratory tests are summarized in Tables 3 and 4, respectively. Age is one of the most important risk factors for colorectal cancer. Therefore, stool donation is recommended from young individuals aged $<45$ years. For donors aged 45 years or above, absence of colorectal cancer should be confirmed by colonoscopy within 3 years of stool donation. Recently, young age onset colorectal cancer comprised up to $10 \%$ of total colorectal cancers in Korea. Colonoscopy might be done optionally for young donors aged $<45$ years. Although colonoscopy can detect undetected colorectal diseases as well as colorectal cancer, medical cost for donor screening should be considered. To date, transmission of colorectal cancer from donor to FMT recipient was not reported worldwide. Tests for transmissible infectious diseases should be included in stool and blood tests. In stool tests, the United States (US) Food and Drug Administration (FDA) recommends additional screening against multidrug-resistant organisms (MDRO), because 2 previous case reports showed FMT-associated extended-spectrum beta-lactamases (ESBL)producing Escherichia coli bacteremia. ${ }^{27}$ Therefore, screening for MDRO was added to other viral, parasitic, and bacterial tests. In a Korean study, only $19 \%$ of the initial donor candidate pool qualified as FMT donors after completion of overall screening. ${ }^{24}$ Although the sample size of this report is much smaller than that of other countries, ${ }^{25,26}$ the results for suitability were acceptable. OpenBiome (Boston, MA, USA) reported that the pass rate of stool donation was only $3 \%$ among 15317 eligible candidates. ${ }^{25}$ In consideration of the extremely low rate of qualification for stool donation, international guidelines for stool banking have reported simplified general blood tests. $^{4}$ 


\section{Preparation for Successful Fecal Microbiota Transplantation Delivery}

\section{Antibiotic Discontinuation}

Statement 5: We suggest that fecal microbiota trans-
plantation recipients with Clostridioides difficile in-
fection discontinue their antibiotics at least 24 hours
before administration of fecal microbiota transplanta-
tion.
(Conditional recommendation, low quality of evidence)

The ultimate goal of FMT is to restore the normal microbiome of the recipients' intestine using healthy donors' stools. Regardless of the type of antibiotics, all antibiotics may adversely affect the FMT material. Therefore, most studies reported a washout period between completing antibiotics and administration of FMT. The duration of the washout period varied from study to study. The shortest and longest durations were 4 hours ${ }^{28}$ and 3 days, ${ }^{29}$ respectively. The majority of studies advocated 24 hours. ${ }^{30-33}$ If possible, we recommend discontinuation of antibiotics for CDI and anti-
CDI antibiotics (metronidazole or vancomycin) at least 24 hours before administration of FMT. However, FMT is not a perfect treatment option for CDI. ${ }^{1,10}$ Considering FMT failure, it is challenging for physicians to cease anti-CDI antibiotics, especially for severe or fulminant $\mathrm{CDI}{ }^{34}$ Also, antimicrobial treatment can be continued when primary infectious diseases were not resolved.

\section{Bowel Lavage}

Statement 6: We suggest bowel lavage before adminis-
tration of fecal microbiota transplantation. The ben-
efits of bowel lavage should be balanced against the
problems faced by patients with swallowing difficulty
or at high risk of aspiration.
(Conditional recommendation, low quality of evidence)

When FMT is performed through colonoscopy, bowel lavage is essential. In addition to facilitating cecal intubation by removing solid material, pre-FMT bowel lavage may have several advantages such as eliminating residual antibiotics, $C$. difficile toxin, spores, and vegetative cells. ${ }^{35,36}$ Bowel lavage may facilitate both engraftment of transplanted microorganisms and safe FMT procedures. ${ }^{3}$ In a meta-analysis, poor bowel preparation was an independent risk factor

Table 3. Clinical Assessment for Donor Candidates

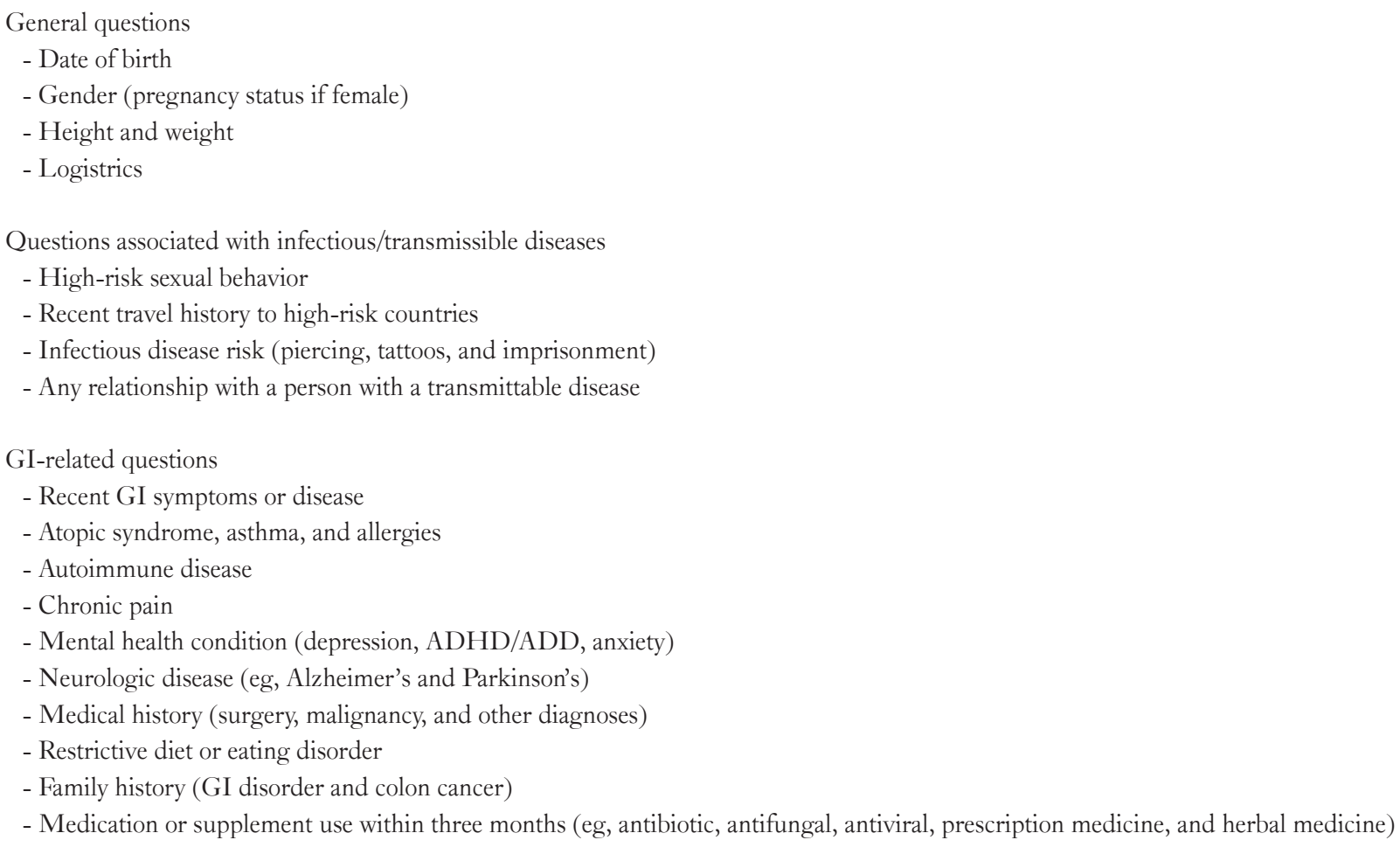

GI, gastrointestinal; ADHD, attention deficit and hyperactivity disorder; ADD, attention deficit disorder. 
Table 4. Serologic Screening and Stool Test for Donor Candidates

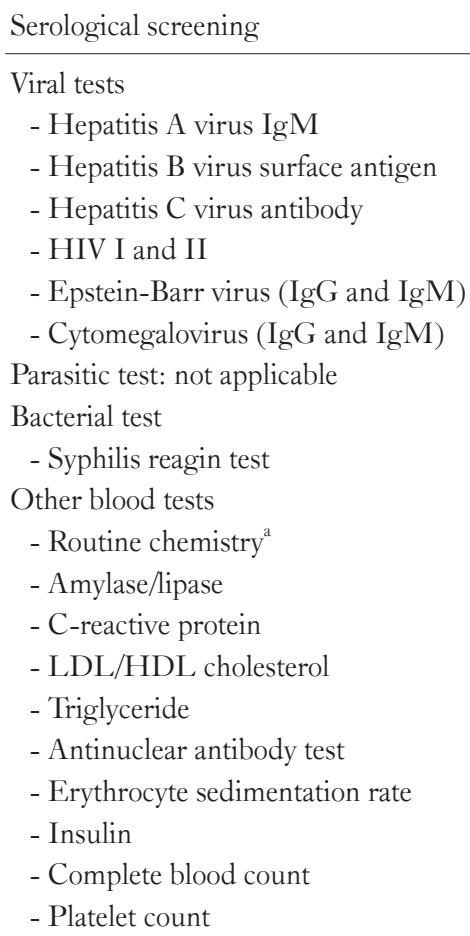

Stool tests

Viral tests

- Viruses associated with diarrhea (RT-PCR): Rotavirus, Norovirus, Adenovirus, Astrovirus

Parasitic tests

- Parasites and ova (multiplex PCR): Ascaris lumbricoides, Cryptosporidium parvum/hominis, Ancylostoma duodenale, Necator americanus, Strongyloides stercoralis, Giardia lamblia, Entamoeba histolytica, Trichuris trichiura, Clonorchis sinensis, Diphyllobothrium latum, Blastocystis hominis

Bacterial tests

- Helicobacter pylori (nested PCR)

- Bacteria associated with diarrhea (PCR): Salmonella spp., Shigella spp., Vibrio spp. Campylobacter spp. (C. jejuni, C. coli),

Yersinia enterocolitica, and Aeromonas spp.

- Multidrug-resistant bacteria: MRSA (Cx), CRE (Cx + PCR), VRE (Cx + PCR), and ESBL-producing Enterobacteriaceae (Cx)

Additional fecal tests

- Fecal white blood cell

- Occult blood

Additional tests

Chest (posteroanterior) radiography

COVID-19 tests (only for pandemic period)

- Nasopharyngeal swab

- Serology for SARS-CoV-2

- Stool testing for SARS-CoV-2

${ }^{a}$ Routine chemistry tests include tests for calcium, inorganic phosphate, glucose, blood urea nitrogen, creatinine, uric acid, total cholesterol, total protein, albumin, alkaline phosphatase, aspartate transaminase, alanine aminotransferase, serum glutamate-pyruvate transaminase, and total bilirubin.

HIV, human immunodeficiency virus; LDL, low density lipoprotein; HDL, high density lipoprotein; RT-PCR, reverse transcription polymerase chain reaction; PCR, polymerase chain reaction; MRSA, methicillin-resistant Staphylococcus aureus; Cx, culture; CRE, carbapenem-resistant Enterobacteriaceae; VRE, vancomycin-resistant enterococci; ESBL, extended spectrum $\beta$-lactamase; SARS-CoV-2, severe acute respiratory syndrome coronavirus 2; COVID-19, coronavirus disease 2019. 
for FMT failure. ${ }^{37}$ Therefore, bowel lavage was performed before FMT using the upper GI tract route, including oral capsules. ${ }^{30,38-40}$ Bowel lavage carries a risk of vomiting and aspiration and may be exempted for patients with high risk of aspiration. ${ }^{41}$ Adoption of bowel lavage should be conducted with caution for patients at high risk of aspiration such as those with (1) swallowing difficulty, (2) ileus, or (3) bedridden status. The benefits of bowel lavage should be weighed against the risks associated with high-risk groups. For safety, polyethylene glycol is recommended for bowel lavage before FMT.

\section{Factors Related to Fecal Microbiota Trans- plantation Procedure}

\section{Stool Amount}

\section{Statement 7: We recommend at least 30-50 g of stool for fecal microbiota transplantation. \\ (Conditional recommendation, low quality of evidence)}

There is limited information to determine the sufficient stool amount for FMT. In a recent systematic review including 168 studies, the infused stool mass ranged from $25 \mathrm{~g}$ to $300 \mathrm{~g}$. ${ }^{9}$ The optimal amount of stool differs among guidelines: $50 \mathrm{~g}$ for the British guideline; ${ }^{3} 30 \mathrm{~g}$ for the European guideline. ${ }^{2}$ The most recently published guideline on stool banking recommends at least $25 \mathrm{~g}$ based on the experience of OpenBiome. ${ }^{4}$

The British guideline recommends using $>50 \mathrm{~g}$ of stool in each FMT preparation based on the finding that the relapse rate after FMT was 4-fold higher when less than $50 \mathrm{~g}$ of stool was administered ( $4 \%$ vs, $1 \%$ for $\geq 50 \mathrm{~g}$ ). ${ }^{3,42}$ A recent meta-analysis also confirmed that a fecal amount $\leq 50 \mathrm{~g}$ was associated with lower efficacy rates after a single infusion in recurrent CDI. ${ }^{43}$ Therefore, it is important to provide a sufficient biomass to restore a healthy microbiota, either by increasing the stool amount in each session, or repeating infusion. In addition, there is wide variability in the microbial content of stool samples between individuals and between different donations, and the stool weight is an imperfect measure of microbiota quantity. ${ }^{44}$ The development of a more objective measure of microbial richness and diversity could be more helpful than setting a specific amount. Regarding stool amount, only 1 study compared the efficacy of FMT between a higher amount and a lower amount of stool. For FMT in patients with IBS, $60 \mathrm{~g}$ of stool showed better efficacy compared with $30 \mathrm{~g}$. ${ }^{45}$

With advancement of stool processing, the quantity of fecal suspension may be decreased while preserving the same quantity of stool. The quantity of stool suspension differed among studies. In the first experience of frozen stool, the quantity of fecal suspension presented was 220-240 mL, which contained $50 \mathrm{~g}$ of stool. ${ }^{46}$ In a Korean stool bank (Microbiotics, Seoul, Korea), $125 \mathrm{~mL}$ of fecal suspension contained $50 \mathrm{~g}$ of stool. In terms of efficacy, the required quantity of stool for FMT should be at least 30-50 g. The stool quantity and method of FMT were heterogenous in studies on FMT for non-CDI. Therefore, it is challenging to define the optimal quantity of stool for non-CDI FMT. The quantity of fecal suspension should be restricted, especially for FMT via the upper GI route. Infusion of a larger volume carries the risk of regurgitation or aspiration for upper GI tract infusions. ${ }^{47}$ The volume of fecal suspension should not exceed 250-300 mL for upper GI infusions. For lower GI tract infusions, a larger-volume infusion may not be related to AEs. Early evacuation of fecal suspension may cause FMT failure or unintended spillage, which is an unpleasant experience for recipients. Antiemetics for upper GI tract infusion and loperamide for colonoscopic infusion may be helpful.

\section{Stool Formulation}

Statement 8: If available, we recommend use of frozen stool for fecal microbiota transplantation, which is manufactured from a stool bank or specialized institute.

(Strong recommendation, high quality of evidence)

Statement 9: Fresh stool, which was rigorously screened, can be used for fecal microbiota transplantation.

(Strong recommendation, high quality of evidence)

Statement 10: A capsular product can be used for fecal microbiota transplantation for individuals without swallowing difficulties.

(Strong recommendation, high quality of evidence)

In the developmental stage of FMT, fresh stool was used for FMT within 6-24 hours of evacuation. In 2012, Hamilton et $\mathrm{al}^{46}$ reported the process of manufacture and efficacy of frozen stool. In the US, frozen stool from a non-profit stool bank is the most commonly used formulation at the moment. ${ }^{48}$ Recently some novel stool formulations have been developed and investigated. ${ }^{49}$ However, the methods to manufacture these novel formulations are mixed and the efficacy of different formulations should be better investigated. Therefore, we focused on comparing the efficacy between frozen 


\begin{tabular}{|c|c|c|c|c|c|c|c|c|c|c|c|}
\hline \multirow{2}{*}{$\begin{array}{l}\text { Study or subgroup } \\
\text { Jiang } 2017\end{array}$} & \multicolumn{2}{|c|}{$\begin{array}{c}\text { Frozen } \\
\text { Events Total }\end{array}$} & \multicolumn{2}{|c|}{$\begin{array}{c}\text { Fresh } \\
\text { Events Total }\end{array}$} & \multirow{2}{*}{$\begin{array}{r}\text { Weight } \\
16.3 \%\end{array}$} & \multirow{2}{*}{$\begin{array}{c}\begin{array}{c}\text { Odds ratio } \\
\text { M-H, fixed, 95\% Cl }\end{array} \\
0.09[0.00,1.76]\end{array}$} & \multicolumn{3}{|c|}{$\begin{array}{c}\text { Odds ratio } \\
\text { M-H, fixed, } 95 \% \mathrm{CI}\end{array}$} & \multirow{2}{*}{\multicolumn{2}{|c|}{$\begin{array}{c}\text { Risk of bias } \\
\text { A B C D E F G } \\
\Theta \Theta \oplus \Theta \oplus \Theta \oplus\end{array}$}} \\
\hline & 20 & 24 & 25 & 25 & & & & & 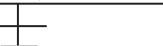 & & \\
\hline Lee 2016 & 57 & 91 & 54 & 87 & $74.9 \%$ & $1.02[0.56,1.88]$ & & & & & $\oplus \oplus \oplus \oplus \oplus \oplus \oplus$ \\
\hline Mathew 2012 & 19 & 21 & 18 & 22 & $6.1 \%$ & $2.11[0.34,12.97]$ & & & & & \\
\hline Satokari 2015 & 22 & 23 & 14 & 15 & $2.7 \%$ & $1.57[0.09,27.21]$ & & & & & \\
\hline Total $(95 \% \mathrm{Cl})$ & & 159 & & 149 & $100.0 \%$ & $0.95[0.56,1.63]$ & & & & & \\
\hline Total events & 118 & & 111 & & & & & & & & \\
\hline $\begin{array}{l}\text { Heterogeneity: } \mathrm{Chi}^{2}= \\
\text { Test for overall effect }\end{array}$ & $\begin{array}{l}=3.34, \mathrm{df} \\
: Z=0.18\end{array}$ & $\begin{array}{l}=3(P \\
(P=\end{array}$ & $\begin{array}{l}P=0.34) \\
0.86)\end{array}$ & $=1$ & & & 0.0 & & 10 & 100 & \\
\hline
\end{tabular}

Risk of bias legend

(A) Random sequence generation (selection bias)

(B) Allocation concealment (selection bias)

(C) Blinding of participants and personnel (performance bias)

(D) Blinding of outcome assessment (detection bias)

(E) Incomplete outcome data (attrition bias)

(F) Selective reporting (reporting bias)

(G) Other bias

Figure. Forest plot of studies comparing frozen stool with fresh stool for fecal microbiota transplantation. M-H, Mantel-Haenszel.

stool and fresh stool for CDI. We included studies that compared frozen and fresh stool. Single-arm studies were not included for analysis. Two RCTs and 2 retrospective studies were included for meta-analysis. ${ }^{46,50-52}$ In total, 159 and 149 patients were included in the frozen and fresh stool group, respectively. The success rate of first FMT was $74.2 \%$ and $74.5 \%$ in the frozen and the fresh stool group, respectively (OR, 0.95; 95\% CI, 0.56-1.63; Figure).

Considering the unsavory aspect of preparing fecal material, frozen stool provided by a certified stool bank is convenient for physicians because it makes the process of recruitment, screening of donor stool, and preparation of a fecal suspension unnecessary. Fresh stool can also be used for FMT if the donor stool is rigorously screened, and stool processing is done in accordance with standard processing.

Capsule FMT is a promising formulation. ${ }^{40,53-55}$ Stool products can be concentrated and prepared as capsules. Initially, capsules contained concentrated liquid and were stored in a refrigerator. Subsequently, lyophilized capsules were developed. Lyophilized capsules can be stored at room temperature, which is more advantageous compared with liquid capsules. The feces can be more concentrated after lyophilization. One capsule of $0.65 \mathrm{~mL}$ contains $1.6 \mathrm{~g}$ of stool. ${ }^{54,56}$ Currently, fresh, frozen, and capsular stool are under investigation for CDI and non-CDI diseases. The resolution of CDI with capsular FMT has been reported as 78-96\%. . $^{40,51,56-58}$ One RCT showed a comparable rate of CDI resolution after single FMT between capsule FMT and colonoscopic infusion (capsule, 96\% [51/53] vs colonoscopy, 96\% [50/52]). ${ }^{40}$ FMT may be performed without the assistance of a physician using capsular stool. In terms of safety, no serious AEs has been reported, including aspiration, to date. We recommend FMT capsules for those without swallowing difficulty. More studies are needed to evaluate the efficacy of FMT treatment using capsule products.

\section{Choice of Infusion Route}

Statement 11: When possible, colonoscopic infusion of fecal suspension into the right colon (terminal ileum or cecum) should be considered initially.

(Strong recommendation, high quality of evidence)

Statement 12: Fecal microbiota transplantation enema can be applied when fecal microbiota transplantation via colonoscopy is not clinically appropriate.

(Conditional recommendation, high quality of evidence)

Statement 13: Fecal microbiota transplantation via upper gastrointestinal tract delivery can also be used where clinically appropriate. Upper gastrointestinal tract should be avoided for patients with high risk of regurgitation or aspiration.

(Conditional recommendation, high quality of evidence)

Fecal delivery can be performed via upper and lower GI tract. Upper GI tract delivery is infusion of donor stools through a gastroscope, nasogastric, nasojejunal, gastrostomy tube, or oral capsule. Lower GI tract delivery is used in the administration of donor stools via colonoscopy, sigmoidoscopy, or retention enema. Choice of infusion route is very important during the FMT procedure. Efficacy and safety need to be considered together when adopting the 
infusion route. In a nationwide registry of the US, 85\% (221/249) of FMT was conducted using colonoscopy. Although the reason for choosing the delivery method was not described in detail, the results of this study provide evidence that colonoscopic infusion is the preferred method for FMT. $^{48}$

We recommend colonoscopic FMT as the preferred infusion route. Several systematic reviews and meta-analyses have revealed that the rate of successful FMT is significantly higher in patients who received FMT via colonoscopy than other delivery methods. ${ }^{1,43,59,60}$ The most recently published meta-analysis reported that the overall cure rate of colonoscopic FMT was $94.8 \%$. In a brief report from OpenBiome, FMT through colonoscopy showed better efficacy compared with the upper GI route. ${ }^{61}$ However, the stool quantity of the 2 delivery routes differed. The provided stool quantity was higher when FMT was performed using colonoscopy than that of the upper GI route. Researchers in the Netherlands pointed out a limitation of the study and questioned the superiority of the lower GI tract route. ${ }^{62}$ Efficacy of FMT is affected by infusion route, bowel preparation, and quantity of stool. To date, there are few studies comparing the efficacy of FMT according to infusion route conducted under the same conditions.

It is recommended that the infusion site of the lower GI access using colonoscopy is the right colon. ${ }^{2,3}$ Guidelines suggest that the preferred location for delivery of FMT is the cecum or terminal ileum, as this procedure may give the highest efficacy. Moreover, the ideal posture of a patient during FMT using colonoscopy has yet to be elucidated. From an anatomical point of view, in order to increase the retention time of fecal suspension in the intestine, it is estimated that the right lateral decubitus posture is more advantageous than the left decubitus posture during and after fecal infusion. For a severely inflamed colon, colonoscopic infusion should be avoided. In such cases, upper GI tract (upper endoscopy or capsule) or, alternatively, the lower GI route (enema or sigmoidoscopic infusion) may be chosen.

Although there is some evidence that FMT enema is less effective than FMT via colonoscopy, ${ }^{10,60}$ FMT enema has the practical benefits of making FMT easily applicable, being less invasive than colonoscopy, and allowing repeated FMT. $^{50,63}$ Where fecal infusion is performed using an enema, it is advised that the patient retains the stool for at least 30 minutes after the fecal suspension infusion and maintains a supine position to minimize bowel movement. ${ }^{2,50} \mathrm{~A}$ recent meta-analysis reported that multiple infusion FMT showed a high success rate, ${ }^{43}$ and thus repeated FMT enema can be considered.

Alternative delivery routes include nasoduodenal tube, ${ }^{64,65}$ upper GI endoscopy, ${ }^{30,41,45,66-68}$ and enteroscopy. ${ }^{47}$ When colono- scopic infusion is not indicated, the upper GI route infusion can be chosen. Patients should remain sitting for few hours after FMT. FMT using the upper GI route may be performed with or without endoscopy (nasogastric tube and nasoduodenal tube). In a patient survey, nasogastric infusion was the most unappealing infusion route for FMT. ${ }^{69}$ Endoscopic infusion may minimize patients' unpleasant sensations more so than methods other than endoscopy. Therefore, endoscopic infusion at duodenal second portion might be recommended for FMT using upper GI route. Theoretically FMT using oral capsule can be regarded as upper GI tract FMT. As discussed above, capsular FMT is favorable among upper GI tract FMT, especially for patients without swallowing difficulty. However, capsular FMT has been regarded as different infusion route, distinct from lower GI tract route or upper GI tract route. The upper GI route is contraindicated for patients with frequent reflux, vomiting, or ileus. When selecting a specific delivery route, an individualized approach is needed that considers both the patient's clinical condition and local expertise.

\section{Safety of Fecal Microbiota Transplantation -}

\section{Statement 14: Patients should be informed of possible adverse events related to procedure and microbiota transfer before fecal microbiota transplantation. \\ (Strong recommendation, low quality of evidence) \\ Statement 15: The overall risk of adverse events of fecal microbiota transplantation via lower gastrointes- tinal tract seems to be lower than that of upper gas- trointestinal tract. \\ (Conditional recommendation, moderate quality of evidence)}

FMT has been reported to be safe for the treatment of recurrent $\mathrm{CDI}$ even in immunocompromised or organ transplantation individuals. ${ }^{39,70,71}$ Serious AEs (SAEs) were observed in $3.2 \%$ of cases, and there was no FMT-related bacteremia after solid organ transplantation. ${ }^{71}$ In a cohort study including 7 CDI patients undergoing hematopoietic stem cell transplantation, FMT was effective ( $86 \%$ of cure rates) and safe. ${ }^{72}$ A systematic review including 303 immunocompromised patients with CDI showed that $87 \%$ had resolution after the first FMT, with $93 \%$ treatment success after multiple FMTs. Reported AEs included 2 FMT-non related deaths, 2 colectomies, 5 bacteremias or infections, and 10 subsequent hospitalizations. ${ }^{73}$ In patients with liver cirrhosis undergoing FMT, the cure rate of CDI was $86 \%$ (54/63 patients), and only 5 
possibly related SAEs occurred..$^{74} \mathrm{~A}$ retrospective study from Israel including 34 patients aged 60 years or older with at least 1 significant comorbidity showed a $90 \%$ clinical improvement with a few SAEs including suspected aspirations, suggesting that FMT is efficacious and safe for elderly patients with underlying illness. ${ }^{75}$

Recently, some fatal AEs were reported and the safety of FMT was questioned. ${ }^{76-78}$ Before FMT, physicians should discuss with their patients or clinical trial participants the risks associated with the procedure. The choice of delivery route needs to be based on the specific clinical situation. AEs of FMT include procedure-related complications and factors related to microbiota transfer.

AEs can be classified as short- or long-term according to the interval between FMT and the occurrence of the AE. Short-term AEs include abdominal pain, diarrhea, flatulence, transient fever, and procedure-related AEs. ${ }^{78}$ A systematic review including 129 studies (4241 patients) from years 2000 to 2020 documented the incidence of FMT-related AEs. The results showed that FMTrelated AEs were identified in 19\% of FMT procedures. Commonly reported immediate AEs after FMT were GI complications, including diarrhea (10\%), abdominal discomfort/pain/cramping (7\%), nausea/vomiting (3\%), and flatulence (3\%). Most of these symptoms were self-limiting and disappeared within a few days. FMT-related SAEs, such as infections and deaths, were reported in 59 patients (1.4\%). Of 5 deaths, 4 were definitely FMT-procedure related, including 1 case of aspiration during sedation and 3 cases of aspiration of the fecal suspension. One was probably FMT-related. ${ }^{79}$

The human gut microenvironment is regarded as an ecosystem. Therefore, transfer of donor microbiota may cause donorhost reaction. Long-term safety or immunologic effects of FMT are relatively uncertain, including the occurrence of latent infections and diseases or conditions related to changes in gut microbiota. One study reported the occurrence of peripheral neuropathy, Sjogren's disease, idiopathic thrombocytopenic purpura, and rheumatoid arthritis. ${ }^{29}$ However, the relationship between FMT and these conditions is not clear. One study found that IBS developed in $4 \%$ of patients after FMT. ${ }^{80}$ Observational studies have demonstrated that FMT is relatively safe during long-term follow up. ${ }^{48,80}$

Transfer of pathogens is one of the serious concerns of FMT. The FDA has reported 6 cases of transfer of $E$. coli from the donor. Of these, 2 patients expired after FMT because of Shigatoxin-producing E. coli. ${ }^{27}$ The donor's stool was not tested for these bacteria at that time. The FDA has requested that testing for MDRO such as carbapenem-resistant Enterobacteriaceae, vancomycin-resistant enterococci, and ESBL producing Enterobacteriaceae should be included in donor screening. ${ }^{81}$ Vigorous donor screening can mini- mize the possibility of transfer of donor's enteric pathogens. Even so, all pathogens cannot be perfectly screened. Therefore, some studies have investigated the efficacy of mixtures of purified bacterial strains, bacterial debris, or novel formulations. ${ }^{82,83}$

In terms of infusion route, nasal stuffiness, sore throat, rhinorrhea, and upper GI hemorrhage, were considered as definitely associated with upper GI routes of administration. ${ }^{76,84,85}$ In addition, some of the common AEs after upper GI administration of FMT are nausea and reflux. ${ }^{65,66}$ When performing FMT via the upper GI tract, one of the main concerns is regurgitation of fecal suspension, which can lead to aspiration pneumonia, which in some cases can be fatal. ${ }^{47,65,76,86}$ Abdominal discomfort, one of the most frequent AEs associated with FMT, was reported in $29.9 \%$ (61/204) of patients after FMT by the upper GI route. For the lower GI route, $13.0 \%$ (56/430) of patients developed abdominal discomfort after FMT. The upper GI route was more likely to develop abdominal discomfort compared with the lower GI route. ${ }^{87}$ The exact mechanism of higher incidence of abdominal discomfort for upper GI infusion was not described. The small bowel is the longest part of GI tract. Despite limited information of the ecosystem of the small bowel, the diversity and density of microbiota in the small bowel are lower than those of the colon. ${ }^{88}$ Large quantities of transferred donor's microbiota may cause small intestinal bacterial overgrowth (SIBO) or adverse interactions between host and donor microbiota. Some cases of SIBO were reported after FMT to date. ${ }^{89}$ SAEs also have been reported regarding colonoscopic infusion. Bowel perforation was reported after colonoscopic delivery. ${ }^{90}$ Deaths related to aspiration was also reported after FMT using colonoscopy. ${ }^{70}$ For patients with high risk of aspiration, the decision to perform FMT should be taken with caution.

Few studies have reported that both upper GI route and lower GI route were safe, with low incidence of AEs. ${ }^{66,91}$ However, in a systematic review the rate of AEs was higher in the upper GI route than in the lower GI tract infusion. ${ }^{79}$ Death related to FMT was reported in 5 cases. Of these, there was 1 case of mortality after colonoscopic infusion.

\section{Follow-up After Fecal Microbiota Transplan- tation}

\section{Statement 16: All fecal microbiota transplantation recipients are required to undergo follow-up in order to assess treatment efficacy, disease recurrence, and possible adverse events.}

(Strong recommendation, high quality of evidence) 


\section{Statement 17: The first week after fecal microbiota transplantation is important to identify symptom reso- lution and short-term adverse events, especially in patients with Clostridioides difficile infection.}

(Strong recommendation, moderate quality of evidence)

\section{Statement 18: The evaluation of primary outcome should be performed at least 8 weeks after fecal mi- crobiota transplantation.}

(Strong recommendation, moderate quality of evidence)

Post-FMT follow-up is required to evaluate efficacy and AEs. However, the follow-up duration and modality vary considerably between studies. The definition of cure in studies targeting recurrent and/or refractory CDI is mostly resolution of symptoms including reduction of stool frequency and improvement of stool consistency, generally over a period of several days. However, outcome parameters including symptom-free days, recurrence of CDI, and AEs have been assessed for more than 8 weeks. The first week after FMT is important to identify short-term AEs and resolution of CDI. In studies selected by our committee, 2 studies, including an RCT, defined primary outcome as a progressive reduction in diarrhea and clinical improvement within 1 week after FMT in patients with $\mathrm{CDI}{ }^{92,93}$ In other studies, the short-term duration of follow-up ranged between 2 weeks and 1 month. ${ }^{71,91,94}$ When FMT is conducted for CDI, symptom resolution was achieved within 1 week of FMT. ${ }^{30,95,96}$ In cases of primary non-response to FMT, repeated FMT may increase the overall cure rate. ${ }^{10}$ Therefore, repeated FMT or rescue therapy, such as oral vancomycin, should be considered at 1 week after FMT for non-response to FMT. When FMT is performed other than for CDI, the primary endpoint may differ among studies. Short-term AEs should be followed at 1 week after FMT.

Symptoms and recurrence of CDI, and AEs after FMT should be monitored over 2 months. The most common followup period of primary outcome in CDI studies was about 2 months. ${ }^{51,64,97,98}$ The studies defined the primary endpoint as clinical resolution and/or absence of CDI recurrence. Early relapse after resolution of $\mathrm{CDI}$ is related to residual toxin from $C$. difficile. Some studies followed the patients up to 3 months. ${ }^{38,40,50,99}$ Other studies used 13 weeks, ${ }^{50} 120$ days, ${ }^{100}$ and 6 months. ${ }^{101,102}$ The British guideline recommends that all FMT recipients should be monitored for at least 8 weeks in total to fully establish efficacy and AEs. ${ }^{3}$ For FMT other than for CDI, the primary outcome will be followed until a predefined period.

\section{Fecal Microbiota Transplantation During COVID-19 Pandemic}

COVID-19 arising from the emergence and spread of severe acute respiratory syndrome coronavirus 2 (SARS-CoV-2) has rapidly progressed into a global pandemic. Emerging evidence shows that SARS-CoV-2 RNA and/or SARS-CoV-2 virus may be found in stools of infected individuals and viral RNA may remain positive in stools even when viral RNA in the respiratory tract is no longer detectable. These results suggest the possibility of transmission of SARS-CoV-2 via a fecal-oral route. ${ }^{103,104}$ International expert panels recommend that at least a nasopharyngeal swab and serology should be considered in potential FMT donors. ${ }^{105}$ Another expert opinion recommends that FMT should be delayed until COVID-19 is better controlled and may be performed only in cases of fulminant CDI without response to maximal combination therapy. ${ }^{106} \mathrm{Ng}$ et $\mathrm{al}^{107}$ reported that a single negative study for stool does not guarantee the absence of SARS-CoV-2, and that testing donors at different time points during the donation period is required. Further research to develop a simple and effective method to find SARS$\mathrm{CoV}-2$ in stool samples is necessary until herd immunity is reached by COVID-19 vaccination. Screening for SARS-CoV-2 should be considered in all donors and recipients if the pandemic persists.

In South Korea, there was an outbreak of Middle East Respiratory Syndrome in 2015. Widespread overseas travel can spread any types of infectious diseases that are rare in Korea across the country. During outbreaks of fatal infectious diseases, stool screening should be performed in a strict manner and FMT may be better performed on a limited basis.

\section{Conclusion}

In this guideline, established by multidisciplinary academic societies, we provide the best practice for FMT in terms of efficacy and safety.

Financial support: This research was supported by a grant of the Korea Health Technology R\&D Project through the Korea Health Industry Development Institute (KHIDI), funded by the Ministry of Health \& Welfare, Republic of Korea (Grant No. HI19C0481, HC20C0099).

\section{Conflicts of interest: None.}

Author contributions: Tae-Geun Gweon have contributed in 
writing and editing the paper as the first authors; Miyoung Choi helped in formulating clinical key questions, conducting relevant literatures search, and mentoring for extensive meta-analyses; Yoo Jin Lee, Kyeong Ok Kim, Sung Kyun Yim, Jae Seung Soh, Seung Young Kim, Jae Jun Park, Seung Yong Shin, Tae Hee Lee, Chang Hwan Choi, Young-Seok Cho, Dongeun Yong, and Jin-Won Chung have contributed in the systematic review, the extraction of recommendations, and writing the paper; Kwang Jae Lee, Oh Young Lee, and Myung-Gyu Choi have contributed as expert panel; and Young-Seok Cho has designed the guideline development as chairman of the Gut Microbiota and Therapy Research Group of KSNM and have revised the manuscript critically.

\section{References}

1. Quraishi MN, Widlak M, Bhala N, et al. Systematic review with metaanalysis: the efficacy of faecal microbiota transplantation for the treatment of recurrent and refractory Clostridium difficile infection. Aliment Pharmacol Ther 2017;46:479-493.

2. Cammarota G, Ianiro G, Tilg H, et al. European consensus conference on faecal microbiota transplantation in clinical practice. Gut 2017;66:569-580.

3. Mullish BH, Quraishi MN, Segal JP, et al. The use of faecal microbiota transplant as treatment for recurrent or refractory Clostridium difficile infection and other potential indications: joint British Society of Gastroenterology (BSG) and Healthcare Infection Society (HIS) guidelines. Gut 2018;67:1920-1941.

4. Cammarota G, Ianiro G, Kelly CR, et al. International consensus conference on stool banking for faecal microbiota transplantation in clinical practice. Gut 2019;68:2111-2121.

5. Ng SC, Kamm MA, Yeoh YK, et al. Scientific frontiers in faecal microbiota transplantation: joint document of Asia-Pacific Association of Gastroenterology (APAGE) and Asia-Pacific Society for Digestive Endoscopy (APSDE). Gut 2020;69:83-91.

6. Haifer C, Kelly CR, Paramsothy S, et al. Australian consensus statements for the regulation, production and use of faecal microbiota transplantation in clinical practice. Gut 2020;69:801-810.

7. Ministry of Health \& Welfare. Korean Academy of Medical Science. Assessment of the quality of clinical practice guidelines in Korea using AGREE 2.0 instrument. 2011. Available from URL: https://www. guideline.or.kr/evaluation/file/AGREE\%202.0_01.pdf (accessed 20 Dec 2021).

8. Schünemann H, Brożek J, Guyatt G, Oxman A. Handbook for grading the quality of evidence and the strength of recommendations using the GRADE approach. 2013. Available from URL: https:/gdt. gradepro.org/app/handbook/handbook.html (assessed 20 Dec 2021).

9. Lai CY, Sung J, Cheng F, et al. Systematic review with meta-analysis: review of donor features, procedures and outcomes in 168 clinical studies of faecal microbiota transplantation. Aliment Pharmacol Ther
2019;49:354-363.

10. Tariq R, Pardi DS, Bartlett MG, Khanna S. Low cure rates in controlled trials of fecal microbiota transplantation for recurrent Clostridium difficile infection: a systematic review and meta-analysis. Clin Infect Dis 2019;68:1351-1358.

11. Surawicz CM, Brandt LJ, Binion DG, et al. Guidelines for diagnosis, treatment, and prevention of Clostridium difficile infections. Am J Gastroenterol 2013;108:478-498.

12. McDonald LC, Gerding DN, Johnson S, et al. Clinical practice guidelines for Clostridium difficile infection in adults and children: 2017 update by the infectious diseases society of America (IDSA) and society for healthcare epidemiology of America (SHEA). Clin Infect Dis 2018;66:e1-e48.

13. Paramsothy S, Kamm MA, Kaakoush NO, et al. Multidonor intensive faecal microbiota transplantation for active ulcerative colitis: a randomised placebo-controlled trial. Lancet 2017;389:1218-1228.

14. Moayyedi P, Surette MG, Kim PT, et al. Fecal microbiota transplantation induces remission in patients with active ulcerative colitis in a randomized controlled trial. Gastroenterology 2015;149:102-109, e6.

15. Rossen NG, Fuentes S, van der Spek MJ, et al. Findings from a randomized controlled trial of fecal transplantation for patients with ulcerative colitis. Gastroenterology 2015;149:110-118, e4.

16. Paramsothy S, Paramsothy R, Rubin DT, et al. Faecal microbiota transplantation for inflammatory bowel disease: a systematic review and metaanalysis. J Crohns Colitis 2017;11:1180-1199.

17. Singh H, Nugent Z, Yu BN, Lix LM, Targownik LE, Bernstenin CN. Higher incidence of Clostridium difficile infection among individuals with inflammatory bowel disease. Gastroenterology 2017;153:430-438, e2.

18. Tariq R, Law CCY, Khanna S, Murthy S, McCurdy JD. The impact of Clostridium difficile infection on mortality in patients with inflammatory bowel disease: a systematic review and meta-analysis. J Clin Gastroenterol 2019;53:127-133.

19. Chen T, Zhou Q, Zhang D, et al. Effect of faecal microbiota transplantation for treatment of Clostridium difficile infection in patients with inflammatory bowel disease: a systematic review and meta-analysis of cohort studies. J Crohns Colitis 2018;12:710-717.

20. Khoruts A, Rank KM, Newman KM, et al. Inflammatory bowel disease affects the outcome of fecal microbiota transplantation for recurrent Clostridium difficile infection. Clin Gastroenterol Hepatol 2016;14:1433-1438.

21. Fischer M, Kao D, Kelly C, et al. Fecal microbiota transplantation is safe and efficacious for recurrent or refractory Clostridium difficile infection in patients with inflammatory bowel disease. Inflamm Bowel Dis 2016;22:2402-2409.

22. Qazi T, Amaratunga T, Barnes EL, Fischer M, Kassam Z, Allegretti JR. The risk of inflammatory bowel disease flares after fecal microbiota transplantation: systematic review and meta-analysis. Gut Microbes 2017;8:574-588.

23. Allegretti JR, Kelly CR, Grinspan A, et al. Inflammatory bowel disease outcomes following fecal microbiota transplantation for recurrent $C$. difficile infection. Inflamm Bowel Dis 2021;27:1371-1378. 
24. Seo HS, Chin HS, Kim YH, et al. Laboratory aspects of donor screening for fecal microbiota transplantation at a Korean fecal microbiota bank. Ann Lab Med 2021;41:424-428.

25. Kassam Z, Dubois N, Ramakrishna B, et al. Donor screening for fecal microbiota transplantation. N Engl J Med 2019;381:2070-2072.

26. Rode AA, Bytzer P, Pedersen OB, Engberg J. Establishing a donor stool bank for faecal microbiota transplantation: methods and feasibility. Eur J Clin Microbiol Infect Dis 2019;38:1837-1847.

27. Food and Drug Administration. Fecal microbiota fortransplantation: safety alert - risk of serious adverse events likely due to transmission of pathogenic organisms. Available from URL: https://www.fda.gov/safety/ medical-product-safety-information/fecal-microbiota-transplantationsafety-alert-risk-serious-adverse-events-likely-due-transmission (assessed 14 Dec 2021).

28. Patel NC, Griesbach CL, DiBaise JK, Orenstein R. Fecal microbiota transplant for recurrent Clostridium difficile infection: Mayo clinic in Arizona experience. Mayo Clin Proc 2013;88:799-805.

29. Brandt LJ, Aroniadis OC, Mellow M, et al. Long-term follow-up of colonoscopic fecal microbiota transplant for recurrent Clostridium difficile infection. Am J Gastroenterol 2012;107:1079-1087.

30. van Nood E, Vrieze A, Nieuwdorp M, et al. Duodenal infusion of donor feces for recurrent Clostridium difficile. N Engl J Med 2013;368:407-415.

31. Zainah H, Hassan M, Shiekh-Sroujieh L, Hassan S, Alangaden G, Ramesh M. Intestinal microbiota transplantation, a simple and effective treatment for severe and refractory Clostridium difficile infection. Dig Dis Sci 2015;60:181-185.

32. Aas J, Gessert CE, Bakken JS. Recurrent Clostridium difficile colitis: case series involving 18 patients treated with donor stool administered via a nasogastric tube. Clin Infect Dis 2003;36:580-585.

33. Kassam Z, Lee CH, Yuan Y, Hunt RH. Fecal microbiota transplantation for Clostridium difficile infection: systematic review and metaanalysis. Am J Gastroenterol 2013;108:500-508.

34. Fischer M, Sipe BW, Rogers NA, et al. Faecal microbiota transplantation plus selected use of vancomycin for severe-complicated Clostridium difficile infection: description of a protocol with high success rate. Aliment Pharmacol Ther 2015;42:470-476.

35. O'Brien CL, Allison GE, Grimpen F, Pavli P. Impact of colonoscopy bowel preparation on intestinal microbiota. PLoS One 2013;8:e62815.

36. Jalanka J, Salonen A, Salojärvi J, et al. Effects of bowel cleansing on the intestinal microbiota. Gut 2015;64:1562-1568.

37. Tariq R, Hayat M, Pardi D, Khanna S. Predictors of failure after fecal microbiota transplantation for recurrent Clostridioides difficile infection: a systematic review and meta-analysis. Eur J Clin Microbiol Infect Dis 2021;40:1383-1392

38. Agrawal M, Aroniadis OC, Brandt LJ, et al. The long-term efficacy and safety of fecal microbiota transplant for recurrent, severe, and complicated Clostridium difficile infection in 146 elderly individuals. J Clin Gastroenterol 2016;50:403-407.

39. Alrabaa S, Jariwala R, Zeitler K, Montero J. Fecal microbiota transplantation outcomes in immunocompetent and immunocompromised patients: a single-center experience. Transpl Infect Dis 2017;19:e12726.
40. Kao D, Roach B, Silva M, et al. Effect of oral capsule- vs colonoscopydelivered fecal microbiota transplantation on recurrent Clostridium difficile infection: a randomized clinical trial. JAMA 2017;318:1985-1993.

41. Gweon TG, Kim J, Lim CH, et al. Fecal microbiota transplantation using upper gastrointestinal tract for the treatment of refractory or severe complicated Clostridium difficile infection in elderly patients in poor medical condition: the first study in an asian country. Gastroenterol Res Pract 2016;2016:2687605.

42. Gough E, Shaikh H, Manges AR. Systematic review of intestinal microbiota transplantation (fecal bacteriotherapy) for recurrent Clostridium difficile infection. Clin Infect Dis 2011;53:994-1002.

43. Ianiro G, Maida M, Burisch J, et al. Efficacy of different faecal microbiota transplantation protocols for Clostridium difficile infection: a systematic review and meta-analysis. United European Gastroenterol J 2018;6:1232-1244.

44. Nicco C, Paule A, Konturek P, Edeas M. From donor to patient: collection, preparation and cryopreservation of fecal samples for fecal microbiota transplantation. Diseases 2020;8:9.

45. El-Salhy M, Hatlebakk JG, Gilja OH, Kristoffersen AB, Hausken T. Efficacy of faecal microbiota transplantation for patients with irritable bowel syndrome in a randomised, double-blind, placebo-controlled study. Gut 2020;69:859-867.

46. Hamilton MJ, Weingarden AR, Sadowsky MJ, Khoruts A. Standardized frozen preparation for transplantation of fecal microbiota for recurrent Clostridium difficile infection. Am J Gastroenterol 2012;107:761767.

47. Link A, Lachmund T, Schulz C, Weigt J, Malfertheiner P. Endoscopic peroral jejunal fecal microbiota transplantation. Dig Liver Dis 2016;48:1336-1339.

48. Kelly CR, Yen EF, Grinspan AM, et al. Fecal microbiota transplantation is highly effective in real-world practice: initial results from the FMT national registry. Gastroenterology 2021;160:183-192, e3.

49. Gweon TG, Na SY. Next generation fecal microbiota transplantation. Clin Endosc 2021;54:152-156.

50. Lee CH, Steiner T, Petrof EO, et al. Frozen vs fresh fecal microbiota transplantation and clinical resolution of diarrhea in patients with recurrent Clostridium difficile infection: a randomized clinical trial. JAMA 2016;315:142-149.

51. Jiang ZD, Ajami NJ, Petrosino JF, et al. Randomised clinical trial: faecal microbiota transplantation for recurrent Clostridum difficile infection - fresh, or frozen, or lyophilised microbiota from a small pool of healthy donors delivered by colonoscopy. Aliment Pharmacol Ther 2017;45:899-908.

52. Satokari R, Mattila E, Kainulainen V, Arkkila PE. Simple faecal preparation and efficacy of frozen inoculum in faecal microbiota transplantation for recurrent Clostridium difficile infection--an observational cohort study. Aliment Pharmacol Ther 2015;41:46-53.

53. Du C, Luo Y, Walsh S, Grinspan A. Oral fecal microbiota transplant capsules are safe and effective for recurrent Clostridioides difficile infection: a systematic review and meta-analysis. J Clin Gastroenterol 2021;55:300-308.

54. Staley C, Hamilton MJ, Vaughn BP, et al. Successful resolution of recur- 
rent Clostridium difficile infection using freeze-dried, encapsulated fecal microbiota; pragmatic cohort study. Am J Gastroenterol 2017;112:940947.

55. Bajaj JS, Fagan A, Gavis EA, Kassam Z, Sikaroodi M, Gillevet PM. Long-term outcomes of fecal microbiota transplantation in patients with cirrhosis. Gastroenterology 2019;156:1921-1923, e3.

56. Youngster I, Russell GH, Pindar C, Ziv-Baran T, Sauk J, Hohmann EL. Oral, capsulized, frozen fecal microbiota transplantation for relapsing Clostridium difficile infection. JAMA 2014;312:1772-1778.

57. Jiang ZD, Jenq RR, Ajami NJ, et al. Safety and preliminary efficacy of orally administered lyophilized fecal microbiota product compared with frozen product given by enema for recurrent Clostridium difficile infection: a randomized clinical trial. PLoS One 2018;13:e205064.

58. Reigadas E, Bouza E, Olmedo M, et al. Faecal microbiota transplantation for recurrent Clostridioides difficile infection: experience with lyophilized oral capsules. J Hosp Infect 2020;105:319-324.

59. Cammarota G, Ianiro G, Gasbarrini A. Fecal microbiota transplantation for the treatment of Clostridium difficile infection: a systematic review. J Clin Gastroenterol 2014;48:693-702.

60. Ramai D, Zakhia K, Fields PJ, et al. Fecal microbiota transplantation (FMT) with colonoscopy is superior to enema and nasogastric tube while comparable to capsule for the treatment of recurrent Clostridioides difficile infection: a systematic review and meta-analysis. Dig Dis Sci 2021;66:369-380.

61. Osman M, O'brien K, Stoltzner Z, et al. Safety and effiacy of fecal microbiota transplantation for recurrent Clostridium difficile infection from an international public stool bank: results from a 2050-patient multicenter cohort. Open Forum Infect Dis 2016;3(suppl 1):2120.

62. Terveer EM, van Beurden YH, van Dorp S, Keller JJ, Kuijper EJ. Is the lower gastrointestinal route really preferred over the upper gastrointestinal route for fecal microbiota transfer? J Clin Gastroenterol 2016;50:895

63. Costello SP, Hughes PA, Waters O, et al. Effect of fecal microbiota transplantation on 8-week remission in patients with ulcerative colitis: a randomized clinical trial. JAMA 2019;321:156-164.

64. Youngster I, Sauk J, Pindar C, et al. Fecal microbiota transplant for relapsing Clostridium difficile infection using a frozen inoculum from unrelated donors: a randomized, open-label, controlled pilot study. Clin Infect Dis 2014;58:1515-1522.

65. van Beurden YH, de Groot PF, van Nood E, Nieuwdorp M, Keller JJ, Goorhuis A. Complications, effectiveness, and long term follow-up of fecal microbiota transfer by nasoduodenal tube for treatment of recurrent Clostridium difficile infection. United European Gastroenterol J 2017;5:868-879.

66. Yang $\mathrm{Z}, \mathrm{Bu} \mathrm{C}$, Yuan $\mathrm{W}$, et al. Fecal microbiota transplant via endoscopic delivering through small intestine and colon: no difference for crohn's disease. Dig Dis Sci 2020;65:150-157.

67. Craven L, Rahman A, Nair Parvathy S, et al. Allogenic fecal microbiota transplantation in patients with nonalcoholic fatty liver disease improves abnormal small intestinal permeability: a randomized control trial. Am J Gastroenterol 2020;115:1055-1065.

68. Camacho-Ortiz A, Gutiérrez-Delgado EM, Garcia-Mazcorro JF, et al.
Randomized clinical trial to evaluate the effect of fecal microbiota transplant for initial Clostridium difficile infection in intestinal microbiome. PLoS One 2017;12:e189768.

69. Zipursky JS, Sidorsky TI, Freedman CA, Sidorsky MN, Kirkland KB. Patient attitudes toward the use of fecal microbiota transplantation in the treatment of recurrent Clostridium difficile infection. Clin Infect Dis 2012;55:1652-1658.

70. Kelly CR, Ihunnah C, Fischer M, et al. Fecal microbiota transplant for treatment of Clostridium difficile infection in immunocompromised patients. Am J Gastroenterol 2014;109:1065-1071.

71. Cheng YW, Phelps E, Ganapini V, et al. Fecal microbiota transplantation for the treatment of recurrent and severe Clostridium difficile infection in solid organ transplant recipients: a multicenter experience. Am J Transplant 2019;19:501-511.

72. Webb BJ, Brunner A, Ford CD, Gazdik MA, Petersen FB, Hoda D. Fecal microbiota transplantation for recurrent Clostridium difficile infection in hematopoietic stem cell transplant recipients. Transpl Infect Dis 2016;18:628-633.

73. Shogbesan O, Poudel DR, Victor S, et al. A systematic review of the efficacy and safety of fecal microbiota transplant for Clostridium difficile infection in immunocompromised patients. Can J Gastroenterol Hepatol 2018;2018:1394379.

74. Cheng YW, Alhaffar D, Saha S, et al. Fecal microbiota transplantation is safe and effective in patients with Clostridioides difficile infection and cirrhosis. Clin Gastroenterol Hepatol 2021;19:1627-1634.

75. Friedman-Korn T, Livovsky DM, Maharshak N, et al. Fecal transplantation for treatment of Clostridium difficile infection in elderly and debilitated patients. Dig Dis Sci 2018;63:198-203.

76. Baxter M, Ahmad T, Colville A, Sheridan R. Fatal aspiration pneumonia as a complication of fecal microbiota transplant. Clin Infect Dis 2015;61:136-137.

77. DeFilipp Z, Bloom PP, Torres Soto M, et al. Drug-resistant E. coli bacteremia transmitted by fecal microbiota transplant. N Engl J Med 2019;381:2043-2050

78. Park SY, Seo GS. Fecal microbiota transplantation: is it safe? Clin Endosc 2021;54:157-160.

79. Marcella C, Cui B, Kelly CR, Ianiro G, Cammarota G, Zhang F. Systematic review: the global incidence of faecal microbiota transplantationrelated adverse events from 2000 to 2020. Aliment Pharmacol Ther 2021;53:33-42.

80. Saha S, Mara K, Pardi DS, Khanna S. Long-term safety of fecal microbiota transplantation for recurrent Clostridioides difficile infection. Gastroenterology 2021;160:1961-1969, e3.

81. Food and Drug Administration. Information pertaining to additional safety protections regarding use of fecal microbiota for transplantation screening and testing of stool donors for multi-drug resistant organisms. Available from URL: https:/www.fda.gov/vaccines-blood-biologics/ safety-availability-biologics/information-pertaining-additional-safetyprotections-regarding-use-fecal-microbiota-transplantation. (assessed 14 Dec 2021)

82. Petrof EO, Gloor GB, Vanner SJ, et al. Stool substitute transplant therapy for the eradication of Clostridium difficile infection: 'RePOOPulating' 
the gut. Microbiome 2013;1:3.

83. Ott SJ, Waetzig GH, Rehman A, et al. Efficacy of sterile fecal filtrate transfer for treating patients with Clostridium difficile infection. Gastroenterology 2017;152:799-811, e7.

84. Vermeire S, Joossens M, Verbeke K, et al. Donor species richness determines faecal microbiota transplantation success in inflammatory bowel disease. J Crohns Colitis 2016;10:387-394.

85. Suskind DL, Singh N, Nielson H, Wahbeh G. Fecal microbial transplant via nasogastric tube for active pediatric ulcerative colitis. J Pediatr Gastroenterol Nutr 2015;60:27-29.

86. Bang BW, Park JS, Kim HK, et al. Fecal microbiota ransplantation for refractory and recurrent Clostridium difficile infection: a case series of nine patients. Korean J Gastroenterol 2017;69:226-231.

87. Wang S, Xu M, Wang W, et al. Systematic review: adverse events of fecal microbiota transplantation. PLoS One 2016;11:e0161174.

88. Kastl AJ Jr, Terry NA, Wu GD, Albenberg LG. The structure and function of the human small intestinal microbiota: current understanding and future directions. Cell Mol Gastroenterol Hepatol 2020;9:3345 .

89. Perler BK, Chen B, Phelps E, et al. Long-term efficacy and safety of fecal microbiota transplantation for treatment of recurrent Clostridioides difficile infection. J Clin Gastroenterol 2020;54:701-706.

90. Obi O, Hampton D, Anderson T, et al. Fecal microbiota transplant for treatment of resistant $C$. difficile infection using a standardized protocol: a community hospital experience. Am J Gastroenterol 2014;109(suppl 2):S629.

91. Gundacker ND, Tamhane A, Walker JB, Morrow CD, Rodriguez JM. Comparative effectiveness of faecal microbiota transplant by route of administration. J Hosp Infect 2017;96:349-352.

92. Ianiro G, Masucci L, Quaranta G, et al. Randomised clinical trial: faecal microbiota transplantation by colonoscopy plus vancomycin for the treatment of severe refractory Clostridium difficile infection-single versus multiple infusions. Aliment Pharmacol Ther 2018;48:152-159.

93. Gallo A, Cancelli C, Ceron E, et al. Fecal calprotectin and need of multiple microbiota trasplantation infusions in Clostridium difficile infection. J Gastroenterol Hepatol 2020;35:1909-1915.

94. Fischer M, Sipe B, Cheng YW, et al. Fecal microbiota transplant in severe and severe-complicated Clostridium difficile: a promising treatment approach. Gut Microbes 2017;8:289-302.

95. Aroniadis OC, Brandt LJ, Greenberg A, et al. Long-term follow-up study of fecal microbiota transplantation for severe and/or complicated Clostridium difficile infection: a multicenter experience. J Clin Gastro- enterol 2016;50:398-402.

96. Khan MA, Sofi AA, Ahmad U, et al. Efficacy and safety of, and patient satisfaction with, colonoscopic-administered fecal microbiota transplantation in relapsing and refractory community- and hospital-acquired Clostridium difficile infection. Can J Gastroenterol Hepatol 2014;28:434438.

97. Pringle PL, Soto MT, Chung RT, Hohmann E. Patients with cirrhosis require more fecal microbiota capsules to cure refractory and recurrent Clostridium difficile infections. Clin Gastroenterol Hepatol 2019;17:791-793.

98. Allegretti JR, Fischer M, Sagi SV, et al. Fecal microbiota transplantation capsules with targeted colonic versus gastric delivery in recurrent Clostridium difficile infection: a comparative cohort analysis of high and lose dose. Dig Dis Sci 2019;64:1672-1678.

99. Mattila E, Uusitalo-Seppälä R, Wuorela M, et al. Fecal transplantation, through colonoscopy, is effective therapy for recurrent Clostridium difficile infection. Gastroenterology 2012;142:490-496.

100. Hota SS, Sales V, Tomlinson G, et al. Oral vancomycin followed by fecal transplantation versus tapering oral vancomycin treatment for recurrent Clostridium difficile infection: an open-label, randomized controlled trial. Clin Infect Dis 2017;64:265-271.

101. Hirten RP, Grinspan A, Fu SC, et al. Microbial engraftment and efficacy of fecal microbiota transplant for Clostridium difficile in patients with and without inflammatory bowel disease. Inflamm Bowel Dis 2019;25:969-979.

102. Tariq R, Saha S, Solanky D, Pardi DS, Khanna S. Predictors and management of failed fecal microbiota transplantation for recurrent Clostridioides difficile infection. J Clin Gastroenterol 2021;55:542-547.

103. Xiao F, Tang M, Zheng X, Liu Y, Li X, Shan H. Evidence for gastrointestinal infection of SARS-CoV-2. Gastroenterology 2020;158:18311833, e3.

104. Wang W, Xu Y, Gao R, et al. Detection of SARS-CoV-2 in different types of clinical specimens. JAMA 2020;323:1843-1844.

105. Ianiro G, Mullish BH, Kelly CR, et al. Reorganisation of faecal microbiota transplant services during the COVID-19 pandemic. Gut 2020;69:1555-1563.

106. Khanna S, Pardi D. Fecal microbiota transplantation for recurrent Clostridioides difficile infection: the COVID-19 era. Am J Gastroenterol 2020;115:971-974.

107. Ng SC, Chan FKL, Chan PKS. Screening FMT donors during the COVID-19 pandemic: a protocol for stool SARS-CoV-2 viral quantification. Lancet Gastroenterol Hepatol 2020;5:642-643. 